\title{
Téoros
}

Revue de recherche en tourisme

\section{La ville et le tourisme}

Relation ancienne, complexité nouvelle et défi conceptuel

\section{Boualem Kadri}

Volume 26, numéro 3, automne 2007

URI : https://id.erudit.org/iderudit/1071011ar

DOI : https://doi.org/10.7202/1071011ar

Aller au sommaire du numéro

\section{Éditeur(s)}

Université du Québec à Montréal

ISSN

0712-8657 (imprimé)

1923-2705 (numérique)

Découvrir la revue

\section{Citer cette note}

Kadri, B. (2007). La ville et le tourisme : relation ancienne, complexité nouvelle et défi conceptuel. Téoros, 26(3), 76-79. https://doi.org/10.7202/1071011ar d'utilisation que vous pouvez consulter en ligne.

https://apropos.erudit.org/fr/usagers/politique-dutilisation/ 


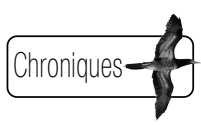

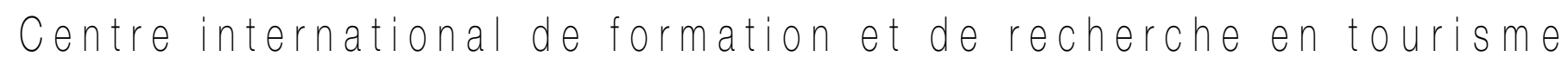

\section{La ville et le tourisme}

\section{Relation ancienne, complexité nouvelle et défi conceptuel}

\section{Boualem Kadri}

Forme ancienne de l'activité touristique, la pratique du tourisme urbain s'est renouvelée d'une manière spectaculaire depuis les années 1980. Ce renouveau a permis à la ville de régénérer son économie (après la crise économique industrielle des années 1970-1980) et d'accroître son attractivité. La relation entre la ville et le tourisme va alors exprimer une réalité nouvelle mais complexe : multiplication des lieux de consommation touristique (culture, nature, divertissement), diversité des activités (affaires, agrément, famille, sport), variabilité des séjours ( $<24$ heures, 1 à 3 jours, > 4 jours), partage de l'espace et des ressources entre les touristes et les résidants. S'exprimant d'abord par ses aspects fonctionnels-attractionnels-récréationnels (Pilette, Kadri, 2005), cette réalité questionne le tourisme urbain dans son aspect conceptuel et dans son apport au processus d'urbanisation. Le présent article vise à montrer la complexité de la relation entre le tourisme et la ville.

\section{La ville et le tourisme : liens historiques anciens, réalité nouvelle complexe}

La ville et le déplacement touristique ont des rapports historiques et dynamiques. Ainsi, si l'on observe un mouvement fait d'attraction-retrait-redécouverte de la ville (Cazes, 2005), on constate que cette dernière tient un rôle structurant dans le processus d'invention du phénomène touristique, étant «à la fois lieu d'impulsion du goût des voyages, mais aussi point de départ, lieu de passage, lieu d'arrivée ou de séjour » (Knafou, 2007 : 9-21).

C'est en Europe que l'invention du tourisme (pratiques, équipements et organisation) prend toute sa signification à travers la transformation des sociétés et des économies (révolutions industrielle et technique, politique, financière) au cours des $X V^{e}-X X^{e}$ siècles. Ainsi, La Renaissance et l'Âge classique montrent une double anticipation dans l'invention touristique: par exemple, les villes d'Italie étalent leur puissance économique et leurs élites sont à la recherche de nouveaux loisirs, telle la réapparition de la villégiature et des résidences autour de la ville, affichant déjà un désir de quitter temporairement cette dernière (Towner, 1996 : 18-19).

Dès les $X V l^{e}$ et $X V I^{e}$ siècles, les guides fixent ce qui doit être vu : par exemple les villes d'Italie et leurs attraits (Rome Florence, Venise) et aussi celles de France, réputées pour leurs institutions du savoir (Montpellier, Poitiers). La période des XVIIIe-XIXe siècles est celle des grandes inventions touristiques: le Grand Tour anglais (début du $\mathrm{XVIII)}$ ), avec la visite des attraits des villes d'Europe par les jeunes aristocrates anglais et l'apprivoisement des territoires limites à la ville (la mer, la campagne, la forêt, la montagne) qui voient l'émergence en Angleterre de la ville d'eau (ex: Bath) et de la station balnéaire (ex: Brighton). Au XIX siècle, la ville industrielle et trop enfumée peut être quittée par les élites urbaines qui investissent «les villes de l'évasion " (villes d'eau, villes côtières) (Pinol, 1991). La ville inquiète, on la quitte, mais elle montre une image double: elle stimule une perception inquiétante par le développement et la démesure, l'apparition de problèmes sociaux, mais elle fascine aussi comme reflet d'un monde plus moderne (Pinol, 1991). Ainsi la ville de la fin du XIXe siècle prépare l'attractivité de la ville de la fin du $X X^{e}$. Déjà, la ville de la fin du XIXe se transforme et introduit un urbanisme souvent monumental, exprimant ainsi sa puissance: Londres innove avec l'Exposition universelle en 1851 et développe la visite urbaine, prélude aux courts séjours urbains du $X X^{e}$, alors que Paris est le laboratoire du loisir de masse, accueillant déjà près de 15 millions de visiteurs (Équipe MIT, 2005 : 57-86). Au Canada, Montréal cherche à innover pour attirer davantage de touristes, notamment durant la saison d'hiver (par ex. : le Carnaval d'hiver), se présentant déjà "comme une ville d'avant-garde, une sorte de fabrique urbaine d'événements et d'attractions » (Pilette et Kadri, $2005: 76)$.

Au XIXe siècle, la forte urbanisation associée à l'industrialisation a donné une perception inquiétante des villes désertées au profit de la nature (mer, campagne, montagne). Au XXe siècle, le développement des stations au bord de la mer se poursuit et attire les plus fortunés: en France, Cannes et Juan les Pins sont vantées par les Américains et les riches urbains dans la période 1910-1930 (Boyer, 1995: 45-47). L'accès au loisir reste encore réservé à des élites et sa diffusion sociale attendra les années 1950. Mais la ville reste encore un pôle émetteur de touristes recherchant la mer et le soleil, et aussi une autre façon de passer des vacances (par ex. : Le Club Med).

À partir des années 1980, la ville et la métropole (re)deviennent une attraction touristique. Cet intérêt renouvelé s'inscrit dans un contexte double : 1) démographique : une grande majorité des personnes (plus de 70 \%) en Europe et en Amérique du Nord habitent les villes, aujourd'hui, et ce phénomène se généralise dans le monde (50\%) ; 2) économique: la crise économique industrielle des années 1970 a créé d'importantes difficultés à la ville (pertes d'emplois, disparition des bases industrielles fonctionnelles, apparition de friches industrielles importantes). Le tourisme contribuera à cette sortie de crise par une régénération de la ville (transformation d'anciens espaces industriels) et la reconstruction d'une nouvelle image. Cette nouvelle attractivité est soutenue par un ensemble de tendances qui favorisent la pratique du tourisme urbain (par ex. : l'intérêt pour la culture et le patrimoine et l'émergence de grands équipements de divertissement). 
La ville postmoderne ne se limite plus à être un lieu de concentration de populations, d'activités (économiques, industrielles et culturelles) et de pouvoirs. Elle joue aussi un rôle de plus en plus important en tant que lieu d'une activité touristique et de loisirs. La grande ville est alors, depuis les années 1980, une destination touristique d'importance (Law, 1996) qui s'est transformée pour devenir attractive.

Le nouvel urbanisme touristique (Page et Hall, 2003) en contexte de postmodernité se distingue par l'importance de la consommation et des expériences dans une perspective de loisir: "What is new, what characterises the 'new urban tourism' is the scale, complexity and diversity of consumption experiences which now exist in urban landscapes built specifically for tourism and leisure. " (Page et Hall, 2003 : 39) La ville des loisirs s'inspirant du modèle des parcs à thèmes propose alors

une urbanité créée de toutes pièces, où le citadin-client peut trouver convivialité et ambiance festive et s'adonner à la consommation du plaisir (consommation à proprement parler, mais aussi loisirs, distractions ou activités préférées), tout en prenant un bain de foule et en se laissant agréablement surprendre par une ambiance de fête bon enfant (Gravari-Barbas, 2006 : 48-56).

Ainsi le tourisme se présente comme un élément moteur de l'aménagement et le stimulateur d'une nouvelle urbanité dans les villes et les métropoles d'aujourd'hui, favorisant l'émergence d'une réalité complexe qui questionne et bouscule la dimension conceptuelle du tourisme urbain.

\section{Le tourisme urbain : un défi conceptuel}

Le renouveau du tourisme urbain est dominé, depuis les années 1980, plus par les interventions sur la ville (mise en spectacle) que par le développement de la réflexion scientifique sur le phénomène lui-même. Pourtant la complexité de ce phénomène caractérisé par les interactions entre le tourisme et la ville devrait stimuler davantage l'intérêt d'un développement théorique et conceptuel nouveau. Certains aspects peuvent exprimer cette lente émergence de la réflexion.

\section{La faible reconnaissance scientifique du tourisme}

L'intérêt pour le tourisme urbain est récent et date de la fin des années 1980 (Ashworth, 1989, cité dans Page et Hall, 1989 ; Cazes et Potier, 1996 et 1998 ; Law, 1996). Toutefois, la lenteur du développement de la réflexion est accentuée par deux éléments importants. D'une part, le statut scientifique mineur du tourisme. En effet, on estime que le développement du tourisme urbain a hérité de certains problèmes du tourisme, notamment son peu d'avancement théorique et conceptuel et son absence d'autonomie par rapport aux autres disciplines des sciences sociales dont il utilise les méthodes et les concepts (Page et Hall, 2003). D'autre part, ce peu de reconnaissance est renforcé par une double négligence des scientifiques, dès la fin des années 1980 : celle des études touristiques pour le contexte urbain et celle des spécialistes de l'urbain pour les fonctions et les activités touristiques dans la ville (Ashworth, 1989, cité dans Page et Hall, 2003). Toutefois, ce sont les spécialistes de la ville qui sont mis à l'index et qui témoignent de ce manque d'intérêt (Cazes, et Potier, 1998). Cet aspect se répercute sur la place de la fonction tou- ristique dans la ville. À cet effet, certains mettent en évidence que le tourisme urbain est perçu comme un élément éphémère, saisonnier, transitoire et non comme un élément important de l'économie urbaine au même titre que les autres fonctions urbaines (Page et Hall, 2003).

Si le tourisme urbain est devenu un stimulant économique et un régénérateur pour les villes, le développement scientifique n'a pas suivi et la compréhension du phénomène reste incomplète (Cazes et Potier,1998). Aussi, la production scientifique réfère à des études descriptives contribuant peu à l'émergence de cadres conceptuels et théoriques (Page et Hall, 2003). Les chercheurs en tourisme sont alors perçus comme prisonniers d'une certaine «confusion » liée aux visions institutionnelles (catégories statistiques) et entretenue par un système (pratiques, institutions et chercheurs) dans lequel le chercheur endosse une certaine responsabilité (Knafou, 2007 : 9-20; Duhamel, 2007 : 351-358).

Le tourisme urbain s'exprime plus par des actions de revalorisation et de promotion que par des données statistiques fiables et riches ${ }^{1}$. Ce manque de données statistiques fiables sur l'activité touristique urbaine réduit l'évaluation de l'importance de la mobilité touristique dans la ville par rapport aux autres formes de tourisme qui présentent de forts pourcentages d'attraction des flux touristiques (Cazes et Potier, 1996). D'une façon générale, les statistiques masquent l'importance du tourisme urbain et l'activité apparaît comme mal comprise, mal maîtrisée (Qui est le touriste urbain ? Que fait-il ?). Le phénomène du tourisme urbain s'avère ainsi être plus complexe que la réalité des stations balnéaires ou de ski (Law, 1996). Cette complexité du phénomène semble avoir retardé l'avancement de la recherche en tourisme urbain caractérisée par la fragmentation et le manque de cohérence, d'où l'urgence de réaliser un cadre conceptuel intégré (Pearce, 2001 : 926-941).

Ces différents aspects nous questionnent sur le concept de tourisme urbain.

\section{Le concept de tourisme urbain : flou et complexité}

La complexité du phénomène touristique urbain semble ne pas se réduire à une définition simple, notamment institutionnelle. Ainsi, en France, la Conférence nationale permanente du tourisme urbain ${ }^{2}$ créée en 1989 , regroupant les villes de 20000 habitants et plus, définit le tourisme urbain de la façon suivante: "Le tourisme urbain est l'ensemble des ressources et activités touristiques implantées en ville, stations mises à part, et proposées aux visiteurs extérieurs. De ce fait il concerne toute personne visitant une ville en dehors de son environnement habituel à des fins de loisirs, d'affaires et autres motifs. " Cette définition est générale (les ressources et les activités), n'accorde pas de place à la dimension temporelle de l'activité (court séjour) et limite l'activité du tourisme urbain à la clientèle extérieure (internationale), en excluant la clientèle locale. Or, dans les métropoles nord-américaines, l'importance de la clientèle des résidants est observée et les pratiques de tourisme urbain sont définies par l'offre (diversifiée) plutôt que par la demande, comme cela se voit en Europe (Pilette et Kadri, 2005 : 10-12).

Une des particularités du tourisme urbain aujourd'hui est qu'il stimule un véritable questionnement concernant le touriste et le phénomène lui-même : le tourisme urbain existe-t-il ? (Page et Hall, 2003 ; Cazes et Potier, 1996 ; Law, 1996). Le tourisme urbain est perçu comme renfermant à la fois un flou conceptuel et une réelle complexité. 
Le flou conceptuel, selon Cazes et Potier (1996), proviendrait de la réunion des deux notions, tourisme et urbain, qui constituent le concept. Selon ces auteurs, l'analyse de l'activité du tourisme urbain se heurte aux définitions normatives reconnues concernant le touriste. Ainsi, les excursionnistes qui séjournent en ville moins de 24 heures ne sont pas considérés comme des touristes au sens de l'Organisation mondiale du tourisme (un touriste est celui qui séjourne 24 heures et plus dans un pays ou une ville), alors que l'excursionniste est un consommateur important dans la ville (visite des musées, utilisation des transports, consommation dans les restaurants). Par ailleurs, en France notamment, l'urbain est défini statistiquement par l'agglomération de 2000 habitants; de ce point de vue, «il est difficile de distinguer la ville du bourg rural, le tourisme urbain du tourisme rural » (Cazes et Potier, 1996 : 13). Ces deux auteurs proposent alors de retenir, du point de vue de l'urbain, la ville de plus de 20000 habitants afin d'intégrer certains équipements et ressources touristiques. Néanmoins, d'autres reconnaissent que cette définition est restrictive et qu'il faudrait inclure certaines villes de moins de 20000 habitants, telles les villes qui ont contribué à l'histoire d'une région (Vighetti, 1994).

Les chercheurs anglo-saxons mettent aussi en évidence ce flou conceptuel d'une manière encore plus prononcée. On y voit dans le concept de tourisme urbain un "concept nébuleux" (a nebulous concept) (Page et Hall, 2003), " un concept chaotique » (Law, 1996) (a chaotic concept) du fait de l'hétérogénéité des espaces urbains (taille, fonctions, et ressources patrimoniales), de l'existence d'une diversité de consommateurs (touristes, résidants locaux et nationaux) et des situations diverses qui mettent en valeur un phénomène plus large et différent des autres formes de tourisme traditionnelles (Law, 1996). Cette situation conceptuelle exprime la complexité de la réalité même de la ville, de la métropole.

Dans la ville postmoderne, la forme spatiale des activités de loisir est représentée par un amalgame complexe de zones urbaines interconnnectées (Page et Hall, 2003). Cette situation montre que la ville postmoderne où le tourisme urbain est fonctionnel s'avère complexe et qu'on ne peut la réduire à un espace de consommation comme ce fût le cas pour la ville historique dans le contexte de la ville moderne et industrielle. Le tourisme urbain postmoderne ne peut être réduit à la ville historique et patrimoniale. De ce fait, le concept de tourisme urbain serait à appliquer au contexte des grandes métropoles où s'expriment la diversité et la multifonctionnalité (Law, 1996). La réalité du tourisme urbain et métropolitain en Amérique du Nord répond à cette nécessité conceptuelle, dans la mesure où ce tourisme se développe par la reconversion d'espaces abandonnés par l'industrie (portuaire, manufacturière) et l'émergence d'une diversité d'équipements (hébergements, sports, congrès, restauration, spectacles) et d'événements (festivals) destinés à une clientèle diversifiée (locale, nationale, internationale) (Pilette et Kadri, 2005). Cette situation montre-t-elle une certaine spécificité du tourisme urbain ?

La problématique de la spécificité du tourisme urbain se retrouve chez plusieurs spécialistes (Cazes, 1994 ; Cazes et Potier,1996; Page et Hall, 2003; Law, 1996). Cazes (1994), par exemple, montre que le tourisme urbain est un phénomène qui invite à poser des « questions dérangeantes ". Cet auteur en identifie cinq qui regroupent les problématiques du tourisme urbain, parmi lesquelles on trouve celle liée à sa spécificité. D'autres auteurs se questionnent sur l'existence du tourisme urbain et du touriste urbain "Does an urban tourism exist? »
(Page et Hall, 2003), «/s there an urban tourism? » (Law, 1996 : 17). Ainsi, le tourisme urbain doit démontrer sa spécificité par rapport aux autres formes de tourisme (mer et soleil, villégiature, montagne). Est-il un complément à ces tourismes ou une forme de consommation particulière avec la ville comme produit? Pour Cazes (1994), cette question est au cœur de plusieurs thématiques entourant le phénomène du tourisme urbain, tels la mobilité stimulée par la multiplication des courts séjours, l'action de revalorisation de la ville, l'accroissement de la consommation culturelle, le développement de la convivialité.

Toutefois, cette question de la spécificité ne doit pas contribuer à diminuer l'importance du phénomène du tourisme urbain et réduire son rôle à un simple fonctionnalisme. Les rapports du tourisme à la ville dépassent cette vision fonctionnaliste (Lussault et Stock, 2007) ; le tourisme s'affiche "comme un élément et un vecteur de l'urbanité comtemporaine " (Lussault et Stock, 2007) et cette diffusion d'urbanité ${ }^{3}$ touche différents espaces, tel celui du rivage touristique (Coeffé, 2007 : 246-255).

Les problématiques de la ville induites par la fonction touristique lancent un défi scientifique aux chercheurs en tourisme contraints de revoir leurs outils conceptuels. Sur ce plan, les géographes ont déjà donné le ton en proposant un renouvellement de l'étude du tourisme et de leur propre discipline: "Ne plus évaluer le tourisme au regard de la géographie mais de partir de l'observation des pratiques " (Lazzaroti, 2003 : 255-277) ; on passe ainsi d'une "géographie du tourisme " à une " approche géographique du tourisme " (Knafou et al., 1997) qui vise « non à affirmer l'autonomie d'une géographie du tourisme, mais à travailler sur le tourisme d'une manière géographique » (Lazzaroti, 2003 : 255-277). Cette vision va-t-elle favoriser le début d'un dialogue entre disciplines nécessaire pour construire une approche scientifique renouvelée du tourisme?

\section{Conclusion}

Le renouveau du tourisme urbain dans les sociétés avancées a eu au moins deux effets importants. D'une part, il a stimulé l'émergence d'une réalité nouvelle et complexe organisée autour des relations entre la ville et le tourisme. Cette situation a permis aux grandes métropoles (par ex. : Londres) de présenter un visage double : celui de la domination (économie et gouvernance mondiales) et celui de la séduction (modèle sociétal) auquel participe la fonction touristique. Le tourisme voit ainsi son statut revalorisé, notamment par les spécialistes de la ville qui le perçoivent non comme spécifique (éphémère, saisonnier), mais comme un "genre commun» lié à la construction du Monde: « II apparaît flagrant que le tourisme n'est pas qu'un produit de l'urbanisation planétaire et de la mondialisation, mais aussi (et surtout) un principe actif de celles-ci. " (Lussault, 2007 : 333-349)

D'autre part, cette réalité complexe bouscule la dimension conceptuelle du tourisme et accroît la nécessité d'une réflexion scientifique sur le tourisme qui s'exprime fortement depuis une décennie 4 . Les géographes, qui investissent le tourisme depuis plusieurs décennies, se distinguent aujourd'hui, notamment les francophones, dans cette remise en question des concepts et des définitions du tourisme. Par exemple, dans leur dernier ouvrage, Les mondes urbains du tourisme, Duhamel et Knafou (2007) ont éliminé l'usage de l'expression « tourisme urbain », perçue comme insatisfaisante pour exprimer une réalité complexe: 
Ce choix se justifie par la découverte que a) le tourisme est intrinsèquement urbain, car fait de citadins - donc pas d'épithète, b) mal nommé, car « tourisme urbain » signifie « tourisme en ville », et ne rend pas compte de la diversité des situations urbaines provoquées par le tourisme : stations touristiques, communautés vacancières, sites touristiques, spots, métropoles touristiques, villes-stations, etc. (Duhamel, 2007 : 351-358).

L'enjeu auquel nous assistons aujourd'hui est celui de la constitution d'une identité scientifique du tourisme qui semble prendre un nouveau départ à partir de l'analyse de la réalité induite par le phénomène touristique urbain. Mais un autre défi surgit, celui de voir comment l'approche disciplinaire, qui domine depuis, laissera place à une démarche transdisciplinaire que l'on estime nécessaire (Dewailly, 2006 ; Lozato-Giotard, 2003). Le rapprochement observé entre certains spécialistes des études urbaines et ceux du tourisme ${ }^{5}$ et la démarche de renouvellement de l'étude proposée par les géographes constituent-ils des prémisses à ce changement?

Boualem Kadri est professeur associé au Département d'études urbaines et touristiques de l'Université du Québec à Montréal.

\section{Notes}

1 L'intérêt d'une bonne observation statistique se fait sentir: l'European Travel Monitor (système d'analyse des comportements touristiques) mène depuis les années 1980 une enquête sur la mobilité interne des Européens. En France, l'INREST (Institut national de recherche sur les transports et leur sécurité) a réalisé dans les années 1990 la première enquête sur les déplacements des Français sur leur territoire. À l'échelle mondiale, l'Organisation mondiale du tourisme (OMT, 2005) a aussi réalisé des enquêtes, en collaboration avec d'autres organismes spécialisés, sur les motivations des touristes urbains européens et internationaux.

2 Site Internet de la Conférence permanente du tourisme urbain en France, [http://www.tourisme-urbain.com], (consulté en août 2007).

3 Pour Michel Lussault et Mathis Stock (2007: 241-245), la question de l'urbanité permet de distinguer les différents espaces où s'insinue le tourisme: "L'urbanité d'un espace donné exprime sa qualité propre, son caractère. Par la saisie de l'urbanité, la différence entre grandes et petites villes, stations touristiques et villes touristiques, agglomérations industrielles et villes administratives, métropoles et mégapoles peut être pensée. Chacun de ces types spatiaux est caractérisé par un niveau d'urbanité différent. "

4 On peut ajouter à la liste bibliographique d'autres auteurs liés à cette réflexion sur le tourisme: Hoerner, J.M. (2003), Traité de tourismologie. Pour une nouvelle science touristique, France, Presses de l'Université de Perpignan; Dewailly, J.M. (2006), Tourisme et géographie: entre pérégrinité et chaos?, Paris, L'Harmattan; Farrel, Bryan H., et L. Twining-Ward (2002), "Reconceptualizing Tourism ", Annals of Tourism Research, vol. 31, $\mathrm{n}^{\circ} 2$, p. 274-275; Tribe, J. (1998), "The Indiscipline of Tourism ", Annals of Tourism Research, vol. 24, n³, p.638-657; Stafford, J. (2003), "La crise de la recherche en tourisme : La solution est dans les problèmes ", Loisir et Société, vol. 26, n¹, printemps, p. 87-95.

5 Ce rapprochement est bien mis en évidence par les éditeurs de Les mondes urbains du tourisme (Duhamel et Knafou, 2007).

\section{Bibliographie}

Boyer, Marc (1995), «L'invention de distinction, moteur du tourisme? Hier et aujourd'hui », Téoros, vol. 14, n² 2, p. 45-47.

Cazes, Georges (1994), «À propos du tourisme urbain: quelques questions préalables et dérangeantes, Les Cahiers Espaces, n 39, décembre, p. 26-30.

Cazes, Georges (2005), "Le tourisme urbain », dans Pierre Merlin et Françoise Choay (dir.), Dictionnaire de l'urbanisme et de l'aménagement, Paris, Presses universitaires de France, p. 891-892.

Cazes, Georges, et Françoise Potier (1996), Le tourisme urbain, Paris, Presses universitaires de France, coll. "Que Sais-je? ».

Cazes, Georges, et Françoise Potier (1998), Le tourisme et la ville: expériences européennes, Paris, L'Harmattan.

Coeffé, Vincent (2007), "L'urbanité cachée du rivage touristique», dans Philippe Duhamel et Rémi Knafou (dir.), Les mondes urbains du tourisme, Paris, Éditions Belin, p. 246-255.

Dewailly, Jean-Michel, (2006), Tourisme et géographie, entre pérégrinité et chaos ?, coll. «Tourismes et sociétés », Paris, Éditions L'Harmattan.

Duhamel, Philippe (2007), «Alors, un énième livre sur le 'tourisme urbain', dans Philippe Duhamel et Rémi Knafou (dir.), Les mondes urbains du tourisme, Paris, Éditions Belin, p. 351-358.

Duhamel, Philippe, et Rémi Knafou (dir.) (2007), Les mondes urbains du tourisme, Paris, Éditions Belin.

Équipe MIT (Mobilité, Itinéraire, Tourismes) (2002), « Un tourisme en nombre », Tourismes 2, Paris, Belin, p. 57-86.

Gravari-Barbas, Maria (2006), La ville à l'ère de la globalisation des loisirs, Éditions Espaces.

Knafou Rémy (2007), "L'urbain et le tourisme. Une construction laborieuse », dans Philippe Duhamel et Rémy Knafou (dir.), Les mondes urbains du tourisme, Paris, Belin, p. 9-21.

Knafou, Rémy et al. (1997), "Une approche géographique du tourisme ", L'Espace géographique, tome 26, n³, p. 193-204.

Law, Christoper Michael (1996), Tourism in Major Cities, London, Routledge.

Lazzaroti, Olivier (2003), "Tourisme et géographie : le grand dérangement ", dans Mathis Stock et al. (coord.), Le tourisme: acteurs, lieux et enjeux, Paris, Belin, p. 255-277.

Lozato-Giotart, Jean-Pierre (2003), Géographie du tourisme: de l'espace consommé à l'espace maitrisé, Paris, Éditions Pearson éducation.

Lussault, Michel (2007), "Le tourisme, un genre commun», dans Philippe Duhamel et Rémi Knafou (dir.), Les mondes urbains du tourisme, Paris, Belin, p. 333-349.

Lussault, Michel, et Mathis Stock (2007), "Tourisme et urbanité», dans Philippe Duhamel et Rémi Knafou (dir.), Les mondes urbains du tourisme, Paris, Belin, p. 241-245.

Organisation mondiale du tourisme et Commission européenne du tourisme (2005), City Tourism \& Culture: the European Experience, Madrid, Organisation mondiale du tourisme et Bruxelles, Commission européenne du tourisme.

Page, J. Stephen, et C. Michael Hall (2003), Managing Urban Tourism, Harlow (Angleterre), Prentice Hall.

Pearce, Douglas, G. (2001), «An Integrative Framework for Urban Tourism Research ", Annals of Tourism Research, vol. 28, n 4, p. 926-946.

Pilette, Danielle et Boualem Kadri (2005), Le tourisme métropolitain. Le cas de Montréal, Québec, Presses de l'Université du Québec.

Pinol, Jean-Luc (1991), Le monde des villes au XIXe siècle, Paris, Hachette.

Towner, John (1996), An Historical Geography of Recreation and Tourism in the Western World: 1540-1940, Toronto, John Wiley \& Sons.

Vighetti, Jean-Bernard (1994), "Qu'est-ce que le tourisme urbain», Les Cahiers Espaces, n³9, décembre, p. 31-35. 